\title{
LXIV. The kinetic theory of gases developed from a new standpoint
}

\section{J.H. Jeans M.A.}

To cite this article: J.H. Jeans M.A. (1903) LXIV. The kinetic theory of gases developed from a new standpoint, Philosophical Magazine Series 6, 5:30, 597-620, DOI: 10.1080/14786440309462965

To link to this article: http://dx.doi.org/10.1080/14786440309462965

曲 Published online: 15 Apr 2009.

Submit your article to this journal 7

Џ Article views: 4

Q View related articles $\square$

Citing articles: 3 View citing articles 5 
TH E

LONDON, EDINBURGH, AND DUBLIN

\title{
PHILOSOPHICAI、 MAGAZINE
}

\author{
AND \\ JOURNAT, OF SCIENCE.
}

[SIXTH SERIES.]

$J U N E 1903$.

LXIV. The Kinetic Theory of Gases developed from a Nev Standpoint. $B y$ J. H. JeANs, M.A., Isaac Newton Student and Fellow of Trinity College, Cambridge*.

\section{Introduction.}

$\S 1.7$ THE aim of the present paper is to develop the Kinetic Theory as far as possible from a purely mathematical standpoint, namely that of abstract dynamies, and in this way to remove certain inconsistencies from the theory.

In the orthodox treatment of the subject a gas is regarded as a collection of similar dynamical systems : these systems interact on one another, and the difficulties of the theory centre largely round the question of determining the occurrence of these interactions. The mothod of the present paper is to regard the whole gas as a single dynamical system. Following this plan we are able to escape all the well-known difficulties-the assumption of a molekular-ungeordnet state, the restriction to infinitely small molecules, \&c.-and may be able ultimately to arrive at a theory which applies to solids and liquids as well as to gases.

\& 2. The basis upon which the orthodox treatment of the subject rests can be shown to be neither à priori logical, nor $\grave{a ̀ ~ p o s t e r i o r i ~ j u s t i f i e d ~ b y ~ s u c c e s s . ~}$

The problem of the Kinetic Theory is, virtually, to follow the motion of a dynamical system starting from an unknown

* Communicated by the Author.

Phil. Mag. S. 6. Vol. 5. No. 30. June 1903. 
configuration. To overcome the difficulty of the initial configuration being unknown we are compelled to regard the whole question as one of probability. Not knowing the positions of individual molecules of the gas, we have to argue : "The probability that there is a molecule of a certain kind within a certain region has such or such a value." The calculation of this value, in the orthodox treatment of the subject, rests upon the "molekular-ungeordnet" assumption of Boltzmann.

The assumption which Boltzmann announces that he is making is that the gas is, and always remains, in a molekularungeordnet state. It does not appear that any strict definition of a molekular-ungeordnet state has ever been attempted. Certainly Boltzmann does not give a definition. Two examples of a geordnet state are given in the Vorlesungen*, and these are of such a special nature that the reader feels convinced that it is legitimate to disregard the genrdnet state altogether. The form in which Boltzmann uses the assumption is somewhat different. This has been pointed out by Burbury, who has clearly stated the assumption in its working form, under the designation of ".Assumption A" $\dagger$.

The effect of this assumption is to enable us to regard certain probabilities at any given instant as independent, and we then assume not only that the probabilities at a later instant are inter se independent, but also that they are independent of the events which took place at any earlier instant. This assumption cannot be logically reconciled with the fact that the motion of the system is continuous in time, i.e., that the events which occur at any instant depend on those which occurred at a previous instant.

$\S 3$. The fundamental assumption, then, cannot be justified ì priori by its consistency. To show that it is not justified it posteriori by its success, it will be necessary to examine some of the consequences of the assumption.

The assumption being granted, it is proved that a certain function $\mathrm{H}$ must continually decrease as the time progresses. From this follows the well-known objection of the reversal of velocitiest. Let $A$ be a system such that the value of $\mathrm{H}$ is $\mathrm{H}_{0}$, and in the course of a small interval of time let it change to a system $\mathrm{B}$, for which $\mathrm{H}$ has the smaller value $\mathrm{H}_{1}$. Then, if we reverse all the velocities in system $B$, we get a second system in which the value of $H$ is still $H_{1}$. The motion of this system must of necessity be through the various configurations which were passed during the change

* Vol. i. p. 20.

+ 'Kinetic Theory of Gases,' p. 9.

$\ddagger$ Boltzmann, Vorlesungen, i. p. 42. 
from $A$ to $B$, and so we arrive at a system which is identical with $A$ except that all the velocities are reversed. For this system the value of $\mathrm{H}$ is $\mathrm{H}_{0}$, and this is greater than $\mathrm{H}_{1}$. How is this to be reconciled with the theorem that $\mathrm{H}$ must always decrease?

Now this theorem of $\mathrm{H}$ decreasing must have been implicitly contained in the equations of motion and the fundamental assumption. The $\mathrm{H}$-theorem points to an irreversible process. This irreversibility cannot have been contained in the equations of motion, for these are essentially reversible in time; it must therefore have been introduced in the molekular-ungeordnet assumption. The view of the present paper, as will appear later, is that the molekularungeordnet assumption is not a true assumption at all, but itmounts to a licence to misuse the calculus of probabilities. The orthodox view is that the decrease in $\mathrm{H}$ is a consequence of supposing the gas to be in a molekular-ungeordnet state, and hence that a gas for which $\mathrm{H}$ increases must be geordnet. By the time this result is reached there seems to be less justification than before for supposing the typical gas to be ungeordnet : it will be seen that to every ungeordnet state there corresponds a geordnet state, so that only one-half at most of all possible arrangements will be ungeordnet. Also of the two corresponding states there does not seem to be :ny reason why one should be labelled ungeordnet rather than the other : in other words it would seem to be just as likely that our results when applied to a real gas should be false as that they should be true. For this reason the assumption of a molekular-ungeordnet state does not seem to be justified hy success.

The present paper contains an outline of a kinetic theory based upon entirely different foundations. This new theory is free from all assumptions, and the arguments are mathematical instead of physical, so that if the reasoning is sound, inconsistencies cannot occur.

\section{The proposed new Basis.}

$\S 4$. A simple illustration will best explain the course of procedure which is to be followed.

Suppose that we are concerned with a series of throws with a die, this die being of the usual type, so that in each throw the chances of each number from one to six being thrown are exactly equal. Suppose that our problem is to find the average value of the throw in an unknown series of throws.

If we consider a series of 10 throws, it can be shown that the "expectation" of the average throw (in other words, the 
average throw averaged over all possible series of 10 throws) is $3 \frac{1}{2}$, but the "expectation" of the difference between this and the average for any single series (in other words, the probable error) is (about) 0.6 . If we consider a series of 1000 throws the expectation of the average throw remains the same, but the probable error is only (about) $0 \cdot 06$. If we pass to the limit, and consider a series consisting of an infinite number of throws, the expectation of the average throw remains $3 \frac{1}{2}$, but the probable error becomes nil.

$\S 5$. This suggests the definite proposition: "The averace value of a throw in an infinite series of throws is $3 \frac{1}{2}$." This proposition does not stand on the same level of absolute truth as the proposition " $2 \times 2=4$," but its truth is sufficient for all practical purposes, and, moreover, it represents the highest level of truth which is attainable in the absence of a definite knowledge of the values of individual throws. The proposition (understood in its proper sense) is not refuted by pointing out that a series such as

$$
1,1,1 \ldots \text {. ad inf. (series A) }
$$

is a possible series, and that the average value in this series is not $3 \frac{1}{2}$ but unity. The reply to this criticism is that the probability is infinitely against a series of random throws being of the form of series $A$, or of any other form for which the proposition is not true ; for a series of random throws it is infinitely more probable that the proposition will be true than that it will be untrue. We may conveniently indicate that a proposition is of this type, by prefixing the words "It is intinitely probable that ..."

$\S 6$. The propositions of the kinetic theory, founded upon the basis which in now proposed, will be of this type; they will state infinite probabilities, and not certainties. The uncertainty as to the positions and velocities of the individual molecules of the gas will correspond to the uncertainty as to the actual values of the throws in the illustrative problem of the dice: the theoretical uncertainty in the final result will replace the uncertainty which enters, in the usual treatment, at the outset by assuming the gas to be ungeordnet. The assumption of an ungeordnet state, as enunciated by Boltzmann*, was obviously intended to exclude special cases analogous to the special case of series $A$. That it does not have this result in the form in which it is used will appear later. But with our present understanding it is quite unnecessary to make any assumption or limitation of this kind, just as in our question of the dice, it was quite unnecessary

* Vorlesungen, i. p. 20. 
to begin by postulating that there should be no "regularity" in the throw of the dice. A limitation of this kind could not, in any case, add anything to the certainty of the ultimate result. Ii may be noticed that the difficulty of giving a precise definition of a molekular-geordnet state of a gas is exactly paralleled by the difficulty which would be experienced in attempting to define "regularity" in the case of the dice.

\section{Outline of the New Theory.}

$\S 7$. Let us now suppose that we are dealing with a great number $\mathrm{N}$ of molecules inclosed in a vessel of volume $\Omega$. We shall consider the simplest case first, and shall accordingly suppose the molecules to be incompressible elastic spheres of the usual type. Each molecule possesses three degrees of freedom, those of molecule A being represented by $x_{a}, y_{a}, \hat{z}_{a}$, the coordinates of its centre. The corresponding velocity coordinates will be denoted by $u_{a}, v_{a}, w_{a^{*}}$. The whole gas, regarded as a single dynamical system, will possess $3 \mathrm{~N}$ degrees of freedom, and its state will be specified by the $6 \mathrm{~N}$ coordinates

$$
u_{a}, v_{a}, w_{a}, x_{a}, y_{a}, z_{n}, \quad u_{b}, v_{b}, w_{b} \ldots \ldots \text {. . . }
$$

Let us imagine a generalized space of $6 \mathrm{~N}$ dimensions. In this space, the system of which the coordinates are given by (1) can be completely and uniquely represented by a single point; namely the point of which the Cartesian coordinates referred to $6 \mathrm{~N}$ definite rectangular axes are given by (1). If our gas is to be entirely inside a certain containing vessel, we shall only need a certain portion of this space, say that bounded by

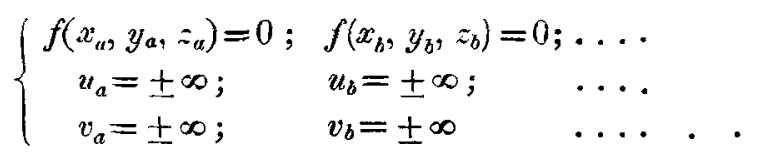

If the molecules are incompressible spheres of radius $R$, we shall not need to consider the possibility of a system in which the centres of any two molecules are within a distance smaller than 2R. We may therefore exclude from consideration all those portions of our generalized space which are bounded by

$$
\left(x_{a}-x_{b}\right)^{2}+\left(y_{a}-y_{b}\right)^{2}+\left(z_{a}-z_{b}\right)^{2}=4 \mathrm{R}^{2}, \quad \text {. . }
$$

and other similar equations, one for every possible pair of molecules. The simplest case of all is that in which $\mathrm{R}=0$; and in this case this last limitation may be disregarded. Taking $\mathbf{R = 0}$ is the sume thing as supposing the diameter to vanish in comparison with the mean free path. 
Any point in the space which remains will represent a single possible configuration of the molecules of the gas. This confignration will, in the course of the natural motion of the gas, give place to other configurations, and these will be represented by new points in the generalized space. The natural motion of the gas may accordingly be represented by the motion of a representative point in the generalized space. Any single point will describe a "path" or "trajectory" in this space, and in this way the whole of the generalized space may be mapped out into trajectories. Since the motion of the gas is completely determined when all the coordinates (1) are given, it follows that through any point there is one and only one trajectory : two trajectories can never intersect. Since the motion of the gas is dynamically reversible, it can be seen that there will be a symmetry in the arrangement of these trajectories. Each trajectory will have an "image" which can be obtained from it by changing the signs of the velocity-coordinates $u, v, u$. So also each point has an image obtained in a similar way. If $\mathrm{P}$ is any point and $\mathrm{P}^{\prime}$ its image, $\mathrm{P}^{\prime}$ represents the system which is obtained by reversing the motion of the system represented by $\mathrm{P}^{*}$.

$\S 8$. We are now going to start an infinite number of our dynamical systems, so as to have systems starting from every conceivable configuration, and try what we can discover about their subsequent motion. $O r$, what comes to the same thing, we are going to imagine the generalized space filled with a continuous fluid, allow this fluid to move along streamlines which coincide with the trajectories already found, and examine the motion of this fluid.

It is obvious that the initial distribution of density in this. fuid may be chosen in a perfectly arbitrary way: all that is necessary is that every point of the generalized space shall be occupied.

We shall find it convenient to choose that the initial distribution of fluid shall be homogeneous. The special advantage

* If we revert to the orthodox standpoint for a moment we see that of all the svstems represented in our generalized space, some will be ungeordnet and some not. We can imagine our generalized space divided into ungeordnet and geordnet regions and points. If $P$ is an ungeor'dnet point, its image $\mathbf{P}^{\prime}$ will, according to Boltzmann (Vorlesungen, i. p. 43) be geordnet, although the converse is not necessarily true. Thus fully half of our generalized space must be geordnet. The conventional treatment of the kinetic theory compels us to assume, that if a trajectory starts from an ungeordnet point, it must pass only through ungeordnet points throughout its whole length. In view of the fact that less than half of the space is ungeordnet, this assumption would seem to be anything. but axiomatic. 
of this choice is that the fluid remains homogeneous throughout the motion. This follows at once from Liouville's theorem**

Not only does the density at every point of the fluid remain constant, but the velocity at this point, being determined solely by the coordinates of the point, will also remain constant. We have therefore to discuss a case of hydrodynamical "steady motion." There is no flow at infinity across the boundary of the generalized space. For at infinity one or more of the velocity-coordinates must be infinite, so that $\mathrm{E}$ the total energy must be infinite; whereas throughout the motion of a point of the fluid $\mathrm{E}$ must remain constant.

\section{Comparison with Orthodox Theory.}

$\S 9$. Let us examine the relation between the procedure now suggested and the usual procedure which rests upon the calculus of probabilities. It may, in the first place, be remarked that a problem of probability has only a definite meaning when a certain amount of knowledge is given and a certain amount withheld. For instance, suppose we have an urn containing a number of masses of different weights. If the weights are known, a question such as "What is the probability that a weight selected at random shall weigh less than an ounce?" has a definite meaning and a definite answer : if the weights are not given, the question is meaningless and has no answer. So also in the Kinetic Theory, it is meaningless to talk about the "probability" of a system being in a specified state: the phrase only acquires a meaning when it is understood that the system is selected at random from a given definite series of systems in different specified states. We shall take this given series of systems to be the series represented by a homogeneous fluid filling our generalized space. On this basis questions of probability will have a definite answer. If we had selected a different series of systems-represented, let us say, by a definite heterogeneous fluid - the answer would be different: if we neglect to specify our series of systems the problem is meaningless, and there is no answer at all.

$\$ 10$. Consider, for instance, the question suggested in $\S 2$. "What is the probability, at a single definite instant, that in the element of volume of which the coordinates lie between $x$ and $x+d x \& c$, there shall be found the centre of a molecule of which the velocities shall lie between $u$ and $u+d u$ \&c.?"

* "On the Conditions necessary for Equipartition of Energy," Phil. Mag. [6], iv. p. 585, equation (7); or J. W. Gibbs, 'Elementary Principles of Statistical Mechanics,' Ch. I. 
With our present conventional basis of probability, we have only to ask "For what proportion of the systems represented in our generalized space is the condition satisfied that there shall be a molecule such that $x$ lies between $x$ and $x+d x \ldots$. \&c., $u$ lies between $u$ and $u+d u$ \&c.?" Now for a certain number of systems this condition will be satisfied by molecule A. These systems will be those for which $x_{a}$ lies between $x$ and $x+d x$; and similar conditions are satisfied by $y_{a}, z_{a}, u_{a}, v_{a}, v_{a}$. These systems together form an element of the generalized space

$$
d x d y d z d u d v d w \iiint_{0} \ldots d x_{b} d x_{c} \ldots d y_{b} d y_{c}, \quad \text {. . }
$$

the integration being with respect to all the $6 \mathrm{~N}$ variables except $x_{a} y_{a} z_{a} u_{a} v_{a} w_{a}$, and extending over all values except such as are excluded by equation (3). Those systems for which the conditions are satisfied by molecule $B$ form an equal and similar element, and so also for every other of the $\mathrm{N}$ molecules. The probability which we require is the ratio of the sum of these $N$ elements to the whole volume of the generalized space, and is therefore

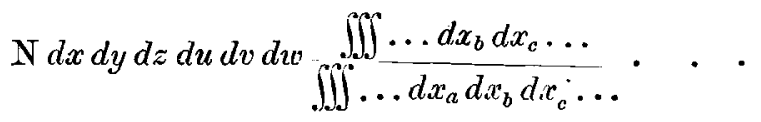

$\S 11$. This is the probability when nothing at all is known about the gas. Let us now suppose that the velocities of the individual molecules are known, and calculate the probability in this case. An element such as (4) will not now be contributed by every molecule: there will be an element contributed by each molecule of which the velocities lie within the assigned limits, and the number of these may be taken to be $\mathrm{N} f(u, v, v) d u d v d w$. The element of volume is not given by expression (4), but by

$$
d x d y d z \iiint_{0} \ldots d x_{b} d x_{\mathrm{e}} \ldots d y_{b} d y_{c}, \quad . \quad \text {. }
$$

the velocity-coordinates not entering into the integration at all. We now find instead of expression (5) a probability

$$
\mathrm{N} f(u, v, w) d x d y d z d u d v d w \frac{\iiint_{0 .} \ldots d x_{b} d x_{e}}{\iiint_{0} \ldots d x_{a} d x_{b} d x_{c} \ldots},
$$

$\S 12$. In the special case of a gas in which the radii of the molecules are very small, the regions which are excluded by 
equations (3) may be neglected, and we may write

$$
\begin{aligned}
& \iiint \ldots d x_{b} d x_{c} \ldots \quad=\prod_{b, e_{,} . .} \iiint d x_{b} d y_{b} d z_{b}=\Omega^{\mathrm{N}-1}, \\
& \iiint \ldots d x_{a} d x_{b} d x_{c} \ldots=\prod_{a, b, c \ldots} \iiint d x_{a} d y_{a}^{*} d z_{a}=\Omega^{\mathrm{N}}
\end{aligned}
$$

for we now have $\iiint d x_{a} d y_{a} d z_{a}=\Omega$, the volume of the containing vessel. Expression (7) now becomes

$$
\frac{\mathrm{N}}{\mathbf{\Omega}} f(u, v, w) d x d y d z d u d v d w . . . .
$$

The first factor is the mean density of molecules in the containing vessel, and the connexion hetween this result and that usually obtained for a gas, whether homogeneous or not, will be obvious.

$\S 13$. Let us now revert to the general result of $\S 11$. We found in expression ( $i$ ) the probability that there should be a molecule (which we may now agree to call $A$ ) of which the coordinates should lie between $x$ and $x+d x$, \&e. Let us now find the probability that, in addition to this, there shall be a second molecule having its six coordinates lying between $x^{\prime}$ and $x^{\prime}+d x^{\prime}, \&$ c. The probability that in addition to the original condition satisfied by $\mathrm{A}$, this second condition shall be satisfied by $B$, can be deduced from expression (7) by limiting the integration in the numerator to values of $x_{b}$ lying between $x^{\prime}$ and $x^{\prime}+d x^{\prime}$, values of $y_{b}$ lying between $y^{\prime}$ and $y^{\prime}+d y^{\prime}$, and so on for $z_{b}, u_{b}, v_{b}, w_{b}$. The probability of which we are in search will be $\mathrm{N} f\left(u^{\prime}, v^{\prime}, w^{\prime}\right) d u^{\prime} d v^{\prime} d w^{\prime}$ times this corrected probability; for this is the number of molecules which can take the part of molecule B. The probability in question is therefore found to be

$\mathrm{N}^{2} f(u, v, w) f\left(u^{\prime}, v^{\prime}, w^{\prime}\right) d x d y d z d u d v d w d x^{\prime} d y^{\prime} d z^{\prime} d u^{\prime} d v^{\prime} d w^{\prime}$

$$
\times \frac{\iiint_{0 .} \ldots d x_{c} d x_{d} \ldots}{\iiint \ldots d x_{a} d x_{b} d x_{c} d x_{d} \ldots} . . . .
$$

$\S 14$. In the special case in which $R=0$ this reduces, by the method of $\$ 12$, to

$\left(\frac{\mathrm{N}}{\bar{\Omega}}\right)^{2} f(u, v, w) f\left(u^{\prime}, v^{\prime}, w^{\prime}\right) d x d y d z d u d v d w d x^{\prime} d y^{\prime} d z^{\prime} d u^{\prime} d v^{\prime} d w^{\prime}$.

$\S 15$. The probability given by expression (10) is exactly that which would be found in a homogeneous gas, with the help of the "molekular-ungeordnet" assumption. The whole supposed point of this assumption is, however, to exclude a 
certain class of systems, whereas it has just been seen that the result arrived at is only true upon the understanding that all conceivable systems-geordnet as well as ungeordnet-are included. It would therefore appear that the effect of this assumption is simply to defeat its own ends : it is brought in ostensibly to make a certain calculation of probability legitimate; whereas in point of fact the calculation is illegitimate (or at any rate cannot be shown to be legitimate) if we adhere to the limitations of the " ungeordnet " assumption, and becomes legitimate as soon as these limitations are ignored.

The calculations which have just been given must, in a logically perfect kinetic theory, replace the "molekularungeordnet" assumption as the justification for treating the possibilities of two molecules having given coordinates as independent events. The fact that the motion of the fluid in our generalized space is steady motion must supply the further justification for supposing these probabilities to remain independent throughout all time. This latter result, it will be noticed, rests on Liouville's theorem, and this in turn rests upon the conservation of energy. There is no justification for supposing probabilities to remain independent when the gas is not a conservative system. It must be noticed that all our results are true only with reference to our arbitrarily chosen basis of probability.

$\S 16$. We have seen that the "ungeordnet" assumption leads to accurate results as regards frequency of collisions; and hence we infer that all results which depend upon this result and upon the dynamies of collisions will be aceurate. The results must, however, be interpreted in a special way. Take, for instance, the H-theorem which deals with a special law of distribution, say $f$. The theorem must not be taken to prove that $d \mathrm{H} / d t$ is negative for all possible systems corresponding to the given $f$, but that the expectation of the value of $d \mathrm{H} / d t$ for a system selected at random from all systems having this $f$ is negative, in other words that the value of $d \mathrm{H} / d t$ averaged over all systems having this given $f$ is negative. There is no justification for confining the theorem to " ungeordnet" systems, and none for stating the proposition to be true of individual systems: it is, so to speak, only true on the average.

$\$ 17$. We are here confronted with a paradox. For each system for which $d \mathrm{H} / d t$ is negative, there will be a second system-the image of the former $(\S 7)$-for which $d \mathrm{H} / d t$ will have an equal but positive value. These two systems must of course be equally included in the average, and since 
the whole number of systems which are included in the average consist of pairs of this kind, it is elear that on the average $d \mathrm{H} / d t$ is zero. We are, in fact, integrating $d \mathrm{H} / d t$ through the region of our generalized space which is given by a specified $f$; this region can be divided into two regions such that one is the image of the other, and the values of $d \mathrm{H} / d t$ in the two regions are equal in magnitude but opposite in sign. The explanation of the apparent paradox will be found in a later section $(\S 32)$.

\section{The Partition of the Generalized Space.}

$\S 18$. We now discuss a number of questions concerning the distribution of systems of various specified kinds in our generalized space.

$\S 19$. The first problem which we shall discuss is concerned with the distribution of density of gas inside the containing vessel.

Let us consider a class of system which we shall call class A. To define it, we imagine the vessel which contains the gas divided into a great number $n$ of "cells," each of equal volume $\omega$, so that $n \omega=\Omega$. These cells are to be denoted by the numbers $1,2,3, \ldots n$. Then a system of class $A$ is defined as one such that $a_{i}$ molecules have their centres in cell $1, a_{2}$ in cell 2 , and in general $a_{\varepsilon}$ in cell $s$, so that of course $\sum_{s=1}^{s=n} a_{s}=\mathrm{N}$.

Denoting individual molecules, as before, by A, B, C..., we see that there will be a certain number of systems represented in our generalized space, such that the molecule A lies in one specified cell, the molecule B in another, and so on for all. It will be easily seen that all systems of this kind vecupy a small continuous element, which we may call the element $\varepsilon$, and which, since there are $\mathrm{N}$ molecules altogether, forms a fraction $(\omega / \Omega)^{N}$ or $n^{-N}$ of the whole. This, it must be noticed, is true whether we consider all systems, or only systems in which the molecules have assigned velocities. Obviously also it is true, if we consider systems in which the kinetic energy has a certain assigned value.

Now if the molecules are distributed in the various cells in such a way that there are $a_{1}$ in cell $1, a_{2}$ in cell 2 , and so on, then all the systems represented by points inside the element $c$ belong to class A. There will be a number of other elements containing only systems of class A; and these will correspond one to each way in which the $\mathrm{N}$ molecules can be distributed into the $n$ cells so that there shall be $a_{1}$ in the first, $a_{2}$ in the 
second, and so on. The number of ways in which this can be done is

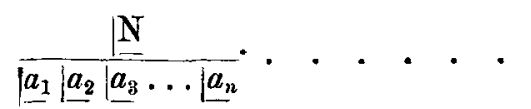

The systems of class $A$ are therefore comprised in this number of elements, each element being of the same size as element $c$. Hence we see that the systems of class A form a fraction $\theta_{a}$ of the whole number of systems, where

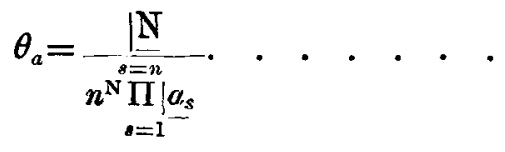

This again is true whether we consider all possible systems or only those systems having a given energy.

$\S 20$. We can evaluate $\theta_{a}$ when $\mathrm{N}$ is large compared with $n$, and there are (in the limit) a very great number of molecules in each of the $n$ cells. We have, when $a_{s}$ is very great, the approximate formula used by Boltzmann*

$$
a_{s}=\sqrt{2 \pi a_{s}}\left(\frac{a_{s}}{e}\right)^{a_{8}}
$$

or taking the logarithm of each side

$$
\log \mid \underline{a_{s}}=\frac{1}{2}(\log 2 \pi+1)+\left(a_{s}+\frac{1}{2}\right)\left(\log a_{s}-1\right) ;
$$

and when $a_{s}$ is very great this assumes the limiting form

$$
\log a_{s}=a_{s} \log a_{s}
$$

an equation in which the difference between the two sides is infinite, but is vanishingly small in comparison with either side. Hence from equation (12),

$$
\begin{aligned}
\log \theta_{a} & =\log |\mathbf{N}-\mathrm{N} \log n-\Sigma \log | a_{s} \\
& =\mathrm{N}(\log \mathrm{N}-\log n)-\Sigma a_{s} \log a_{s} .
\end{aligned}
$$

Now $\mathrm{N}=\Sigma a_{s}$, and we may write $\mathrm{N} / n=a_{0}$, where $a_{0}$ is the mean number of molecules in each cell. Making these substitutions, our equation becomes

$$
\log \theta_{a}=\Sigma a_{s} \log a_{0}-\Sigma a_{s} \log a_{s}=-\Sigma a_{s} \log \left(\frac{a_{s}}{a_{0}}\right) .
$$

The right-hand member of this equation is, in general, an

*Vorlesungen, i. p. 41. 
infinite quantity of the order of $\mathrm{N}$, and is for this reason difficult to manipulate. We accordingly introduce a new quantity $\mathrm{K}_{a}$ defined by

$$
\mathrm{K}_{a}=\Sigma \frac{a_{s}}{\mathrm{~N}} \log \left(\frac{a_{s}}{a_{0}}\right) ; . . . . .
$$

so that $\mathrm{K}$ is, in general, finite; and we have

$$
\theta_{a}=e^{-\mathrm{NK}_{a_{*}}} \text {. . . . . . . }
$$

$\S 21$. But now the different classes of systems may be represented in a new generalized space-the subsidiary space, as we may call it. This space is to have $n-1$ dimensions, corresponding to $n$ variables $\xi_{1} \xi_{2} \ldots \xi_{n}$ subject to $\Sigma \xi=N$. A system of class $A$ is represented by the point

$$
\xi_{1}=a_{1}, \quad \xi_{2}=a_{2}, \ldots \xi_{s}=a_{s} . . . . .
$$

which we shall call the point $A$.

The various classes may therefore be represented by the intersections of $n$ systems of paraliel lines drawn at unit distance apart. Further, we can represent the different, systems which occurred in our original generalized space in the subsidiary space, by placing an imaginary mass $\theta_{a}$ at the point $\mathrm{A}, \theta_{b}$ at $\mathrm{B}$, and so on.

We now pass to the limit and take $N=\infty$. The values of $\xi_{1}, \xi_{2} \ldots$ (or what is the same thing $a_{1}, a_{2} \ldots$ ) will now be infinitely great, and we accordingly take the unit of length in our subsidiary space to be infinitely small.

We can now replace the distribution at points by a continuous volume-distribution. The distribution $\theta_{\alpha}$ at point $\mathrm{A}$ is now to be replaced by a density $\rho$ spread through the rectangular unit element of which $A$ is the centre such that the aggregate density in this unit element is $\theta_{a}$. It is obviously possible (equation 14) to take

$$
\rho=e^{-\mathrm{N} \overline{\mathrm{K}}}
$$

as the general continuous distribution, where $\overline{\mathrm{K}}$ may be chosen so that it is continuous, and becomes identical with $K_{a}$ at $A$, $K_{b}$ at $B$, \&c. All the systems in our original space may in this way be represented in our subsidiary space.

The number of systems of any specified type in our original generalized space (the total being taken as unity) is therefore equal to the number of the same type in the subsidiary space, and this

$$
=\sum_{A, B, \ldots} \theta_{a}=\iiint \cdots \rho d \xi_{1} d \xi_{2} \ldots
$$


In particular, the number for which $K$ (defined by equation 13) lies between $K_{1}$ and $K_{2}$ will be

$$
\iiint_{\overline{\mathrm{K}}=\mathrm{K}_{\mathrm{l}}}^{\overline{\mathrm{K}}=\mathrm{K}_{2}} \ldots e^{-\mathrm{N} \overline{\mathrm{K}}} d \xi_{1} d \xi_{2} \ldots . . .
$$

where the integration extends through those parts of the subsidiary space for which $\overline{\mathrm{K}}$ lies between $\mathrm{K}_{1}$ and $\mathrm{K}_{2}$.

$\S 22$. Now in the subsidiary space $\overline{\mathrm{K}}$ will be found to have a minimum value. In equation (13), let us treat $\left(\frac{a_{s}}{a_{\mathrm{a}}}\right)$ as a continuous variable, and replace it by $\theta$. We then have

$$
\overline{\mathrm{K}}=\mathrm{K}=\sum_{s} \frac{a_{0}}{\overline{\mathrm{N}}} \overline{a_{s}} \log \left(\frac{a_{s}}{a_{0}}\right)=\frac{1}{n} \Sigma_{s} \theta_{s} \log \theta_{s} . \quad .
$$

The values of $\theta$ are limited by the condition

$$
\Sigma \frac{a_{8}}{a_{0}}=\frac{N}{a_{0}}=n
$$

or what is the same thing, by

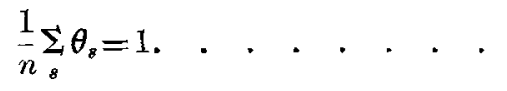

For small variations in $\theta$, the condition that $\overline{\mathrm{K}}$ shall be a minimum is seen by variation of equations (17) and (18) to be

$$
\underset{n}{1} \sum_{s}\left\{1+\log \theta_{s}+\lambda\right\} \delta \theta_{s}=0, . . . .
$$

where $\lambda$ is an indeterminate multiplier. We must therefore have

$$
1+\log \theta_{s}+\lambda=0 \quad \text {. . . . . }
$$

for all values of $s$, and hence $\theta_{s}=$ constant. From equation (18) we see that this constant value must be $\theta_{s}=1$. Thus when we regard $a_{s} / a_{0}$ as a continuous variable the minimum occurs when $a_{1}=a_{2}=\ldots=a_{s}=\ldots=a_{0}$. When $a_{s}$ is very great, and only capable of integral values, the minimum still occurs for values such that, except for infinitesimally -mall quantities,

$$
\frac{a_{1}}{a_{0}}=\frac{a_{2}}{a_{0}}=\ldots=\frac{a_{s}}{a_{0}}=\ldots=1 . . . \text {. . . }
$$

The minimum value of $\bar{K}$ is found to be $\bar{K}=0$, except for an infinitesimally. small quantity : it does not, however, follow that $\mathrm{N} \overline{\mathrm{K}}=0$. The minimum value of $\overline{\mathrm{K}}$ subject to 
$a_{1}, a_{2}$, \&c., being integral, will, however, be greater than the unrestricted minimum for $\overline{\mathrm{K}}$, so that the infinitesimal value of this former minimum will be positive.

Hence it follows that, except at one point in our subsidiary space and points which are in its immediate neighbourhood, $e^{-\mathrm{N} \overline{\mathrm{K}}}$ is vanishingly small. The density at this point is therefore infinite in comparison with that elsewhere.

$\S 23$. There now three possibilities between which we have to discriminate. If we draw a small region in our subsidiary space, inclosing the minimum value of $\mathrm{K}$, the density integrated throughout this small region may be either vanishingly small or finite or infinitely great in comparison with the integral density taken throughout the remaining space. It will be found that the last possibility is the true one.

To prove this, we expand $\overline{\mathrm{K}}$ in the neighbourhood of the minimum value $\bar{K}=0$. We have (equation 17 )

$$
\overline{\mathrm{K}}=\frac{1}{n} \sum_{s} \frac{a_{s}}{a_{0}} \log \frac{a_{s}}{a_{0}} \text {. . . . . . }
$$

If we put $a_{s}=a_{0}+\epsilon_{s}$, where $\epsilon_{s} / a_{0}$ is small, we get

$$
\begin{aligned}
\frac{a_{s}}{a_{0}} & =1+\frac{\epsilon_{s}}{a_{0}} \\
\log \frac{a_{s}}{a_{0}} & =\frac{\epsilon_{s}}{a_{0}}-\frac{1}{2}\left(\frac{\epsilon_{s}}{a_{0}}\right)^{2}+\ldots,
\end{aligned}
$$

whence, since $\sum_{s} \epsilon_{s}=0$,

$$
\overline{\mathrm{K}}=\frac{1}{2 n} \sum_{s}\left(\frac{\epsilon_{s}}{a_{0}}\right)^{2} \cdot \cdot \cdot \cdot \cdot \cdot \cdot .
$$

We may now suppose the small region inclosing the minimum value of $\overline{\mathrm{K}}$ to be bounded by $\overline{\mathrm{K}}=\kappa$, where $\boldsymbol{\kappa}$ is a small positive quantity, and the equation of this boundary in rectangular coordinates will, by equation (23), be

$$
\Sigma\left(\frac{\varepsilon_{s}}{a_{0}}\right)^{2}=\Sigma n \kappa
$$

Hence we see that $\iiint_{. . . d \xi_{1}} d \xi_{2} \ldots$ taken inside the region $\mathrm{K}<\kappa$, is proportional to $\kappa^{n-1}$ so long as $\kappa$ is small. $\mathrm{By}$ differentiation, we find that the value of the integral from $\mathbf{K}$ to $\mathrm{K}+d \mathrm{~K}$ is proportional to $\mathrm{K}^{n-1} d \mathrm{~K}$ so long as $\mathrm{K}$ is small. Hence, except for a multiplying factor, the integral (16) may be written in the form

$$
\int_{\overline{\mathrm{K}}=\mathrm{K}_{1}}^{\overline{\mathrm{K}}=\mathrm{K}_{2}} e^{-\mathrm{N} \overline{\mathrm{K}}} \overline{\mathrm{K}}^{n-1} d \overline{\mathrm{K}} \ldots . . . .
$$


so long as $K_{1}, K_{2}$ are both small. We now see that in this case the whole value of the integral arises from the contribution supplied by an infinitesimal range of values of $\overline{\mathrm{K}}$ near to and including the lower limit. It is obvious that the proof can be extended so as to include all values of $\bar{K}$ whether great or not.

\$24. Returning to the original generalized space, the following propositions will now be seen to be true :-

(i.) All except an infinitesimally sinall fraction of the whole space has a value. of $\mathrm{K}$ which $<\epsilon$, where $\epsilon$ is a small positive quantity, and this is true however small $\epsilon$ may he.

(ii.) $\mathrm{K}$ is positive at every point-either finite or vanishingly small-and the mean value of $K$ averaged throughout the whole space is vanishingly small.

(iii.) Of that part of the space for which $\mathrm{K}>\mathrm{K}_{0}$, where $K_{0}$ is some finite positive quantity, all except an infinitesimal fraction of the whole has a value of $\mathrm{K}$ which $<\mathrm{K}_{0}+\epsilon$, where $\epsilon$ is a small positive quantity, and this is true however small $\epsilon$ may be.

$\$ 25$. Let us now suppose that the edges of our cells are so great compared with the average distance between two molecules that we may legitimately regard the number of molecules in each cell as infinitely great, but that at the same time these edges are so small compared with the scale on which the density of the gas varies, that we may regard the density as constant over a large number of adjacent cells. If the cells can be constructed so that these suppositions are simultaneously possible, it will be possible to give a definition of the density at a point in the gas which shall be logically sound-we define the density at any point $P$ as the number (or mass) of molecules in the cell which contains $P$, divided by the rolume of this cell.

Let us denote the density at a point by $\rho$ and the meandensity of the gas by $\rho_{0}$. By hypothesis, $\rho$ is appreciably constant throughout a cell. An integral such as $\iiint \rho d x d y d z$ taken throughout a cell may accordingly be replaced by $\rho \omega$. The value of $\rho / \rho_{0}$ in the $s^{\text {th }}$ cell may be replaced by $a_{s} / a_{0}$, when the system is of class $A(\$ 19)$.

We now see that instead of defining $K$ by equation (13) we may suppose it defined by

$$
\mathrm{K}=\frac{1}{n} \Sigma \frac{\rho}{\rho_{0}} \log \left(\frac{\rho}{\rho_{0}}\right)
$$

where the summation extends throughout all the cells, and 
if we replace $\rho$ by $\frac{1}{\omega} \iiint \rho d x d y d z$, this becomes

$$
\mathrm{K}=\frac{1}{\Omega} \int 1 \int_{\rho_{0}}^{\rho} \log \left(\frac{\rho}{\rho_{0}}\right) d x d y d z . \quad . \quad .
$$

We have now obtained a value of $K$ which is dependent only on the positions of the molecules, being entirely independent of the way in which the gas was divided up into cells. The propositions stated in $\$ 24$ are therefore independent of the arrangement of cells.

$\S 26$. Equation (21) shows that the minimum value of $K$ is given when $\rho=\rho_{0}$ everywhere ; i.e., when the density is constant throughout the gas. Proposition (i.) of $\$ \mathbf{2 4}$ may therefore be taken to state that if a point be selected at random in our generalized space, it is infinitely probable that the corresponding system will be one for which $\rho$ is constant everywhere.

This is not difficult to understand. Selecting a point at random in our generalized space is equivalent to placing $\mathbf{N}$ molecules at random at points inside the containing vessel. The fundamental principles of the theory of probability lead us to expect that it will be infinitely probable that the distribution of gas will be uniform.

$\S 27$. We have discussed the distribution of $x, y, \approx$ coordinates: the distribution of $u, v, w$ coordinates can be treated in the same way. In this case, however, what is required (for reasons which will appear later) is not a knowledge of the partition of the whole space, but only of that part of it for which the kinetic energy has an assigned value E. This is the part given by the equation

$$
\frac{1}{2} m \sum_{a, b, \ldots}\left(u_{a}{ }^{2}+v_{a}{ }^{2}+w_{a}^{2}\right)=\mathrm{E} . \quad . \quad .
$$

Selecting a point at random from this part of our space, is not equivalent to distributing velocities at random to the $\mathrm{N}$ molecules, but is equivalent to distributing $3 \mathrm{~N}$ velocities $\left(u_{a}, v_{a}, w_{a}, u_{b}, v_{b}, \& c\right.$. $)$ about a mean value $2 \mathrm{E} / 3 \mathrm{mN}$. We shall therefore not expect the resulting distribution to be uniform, but shall expect the velocities to be grouped about this mean value according to the law of trial and error.

We could treat the whole question on the lines on which the former question was treated. We should find that instead of the old function $\mathrm{K}$ defined by equation (25), we should have a function $\mathrm{H}$ defined by

$$
\mathrm{H}=\iiint f \log f d u d v d v
$$

where $f$ is a function of $u, v, w$ such that the number of Phil. Mag. S. 6. Vol. 5. No. 30. June 1903. 
molecules of which the velocities lie between $u$ and $u+d u$, \&c. is $\mathrm{N} f(u, v, w) d u d v d w$. We find that $\mathrm{H}$ has a minimum value for the region defined by equation $(26)$, and we find that the propositions of $\S 24$ apply equally, mutatis mutandis, to this case.

The minimum value of $\mathrm{H}$ and the corresponding law of distribution can be found by the variation of equation (27), keeping $f$ subject to

$$
\begin{aligned}
& \iiint f d u d v d u=1, \ldots . \\
& \iiint \frac{1}{2} m\left(u^{2}+v^{2}+w^{2}\right) f d u d v d w=\frac{\mathrm{E}}{\mathrm{N}} .
\end{aligned}
$$

The resulting equation is

$$
\iiint\left(1+\log f+\lambda+\frac{1}{2} \mu m\left(u^{2}+v^{2}+w^{2}\right)\right) \delta f d u d v d w=0, \text {. }
$$

where $\lambda, \mu$ are indeterminate multipliers. The solution is

$$
1+\log f+\lambda+\frac{1}{2} \mu m\left(u^{2}+v^{2}+w^{2}\right)=0 ; . \quad .
$$

or, changing $\lambda, \mu$ for new constants,

$$
f=\mathrm{A} e^{-h m\left(u^{2}+v^{2}+x^{2}\right)}, \quad . \quad . \quad . \quad .
$$

the well-known law of Maxwell and Boltzmann.

$\S 28$. This law gives $f=0$ when $u, v$, or $w$ becomes infinite. There is therefore the difficulty that if we divide all possible velocities into "cells" in the manner of $\S 19$, the number of molecules in some of these cells cannot be treated as infinitely great. The difficulty is best met by taking a definite velocity $\mathrm{V}$, such that the molecules of which the velocities do not satisfy

$$
u<\mathrm{V}, \quad v<\mathrm{V}, \quad u<\mathrm{V}, \quad . \quad . \quad .
$$

form an infinitesimal fraction of the whole. If the velocities which satisfy (33) can be partitioned into cells in the manner of $\S 19$, so as to satisfy the conditions of $\S 25$, there is no further difficulty, and equation (32) gives the law for velocities which satisfy (33). The law has no meaning for velocities which do not satisfy (33). It is obvious, for instance, that the law given by equation (32) does not impose any upper limit whatever on the possible values of $u, v$, and $v$ for a single molecule, whereas in point of fact such a limit is definitely imposed by equation (26).

$\S 29$. We have investigated the law of partition of the coordinates $x, y, z$ and of the coordinates $u, v, w$ separately. They could have been quite easily investigated together as follows : 
Assume the number of molecules having coordinates lying between $x$ and $x+d x, \ldots u$ and $u+d u \ldots \& c$., to be

$$
\mathrm{N} f(x, y, z, u, v, w) d x d y d z d u d v d w, .
$$

and introduce a new function $\mathrm{H}$ defined by

$$
\mathrm{H}=\int\left[\int\right] \int \rho f \log f d x d y d z d u d v d w \text {. }
$$

Then it will easily be seen that the propositions of $\$ 24$ apply to the region of our generalized space for which the total energy is $E$, provided we replace $K$ by $H$, and instead of $\mathrm{K}=0$, write $\mathrm{H}=\mathrm{H}_{0}$, where $\mathrm{H}_{0}$ is the minimum value of $\mathrm{H}$ defined by equation (35), when $f$ is subject to the conditions

$$
\begin{aligned}
& \iiint \iiint f d x d y d z d u d v d v=1, \quad . \quad . \quad . \quad . . . \\
& \iiint \iiint \frac{1}{2} m\left(u^{2}+v^{2}+v^{2}\right) f d x d y d z d u d v d w=\mathrm{E} .
\end{aligned}
$$

The resuling law is found to be

$$
f(u, v, w, x, y, z)=\mathrm{A} e^{-h m\left(u^{2}+v^{2}+w^{2}\right)}, . \quad .
$$

giving uniformity of density for all values of $x, y, z$ and Maxwell's law of distribution of velocities in a single equation.

\section{The Normal State.}

$\S 30$. When the $6 \mathrm{~N}$ coordinates of the molecules of a gas are such that $H$ has its minimum value, or a value which differs from this minimum by an infinitesimally small amount, the gas will be said to be in its "normal state." For gases in states other than the normal, the difference between the value of $\mathrm{H}$ for the gas and the minimum possible value corresponding to the same energy, may be taken to supply a measure of the divergence of the gas from the normal state. by

We now find that proposition (i.) of $\$ 24$ may be replaced

(iv.) All except an infinitesimally small fraction of the whole of the generalized space represents gases which are in their normal states.

$\$ 31$. Let us now examine what becomes of the two remaining propositions.

Suppose that we start the gas from a configuration about which nothing is known except that the total energy is $\mathrm{E}$ (speaking physically, we have a gas of which the temperature and pressure are known), then the representative point may be supposed to be selected at random from that part of our generalized space for which the energy is $\mathrm{E}$. Suppose we 
try to calculate the average value of $H$ throughout the motion of the gas. Since nothing is known about the initial configuration, nothing is known as to which trajectory the representative point is describing. Thus, relatively to the knowledge which we possess, and to our arbitrary basis of probability, the representative point is at any instant equally likely to be at any point of our generalized space for which $\mathrm{E}$ has the assigned value. Hence the expectation of the average value of $H$ throughout the motion (lasting through any interval we please) is exactly the same as the average value of $\mathrm{H}$ throughout the whole of the region for which $\mathrm{E}$ has the assigned value, and therefore is equal to the minimum value of $\mathrm{H}$ throughout this region. It follows that all the physical properties of the gas (i.e., properties which depend, firstly, only on the statistical law of distribution, and not on the individual molecules, and, secondly, only on the values integrated through an interval of time, and not on values at any single instant, e.g., the pressure of the gas) may be calculated on the supposition. that the gas is in the normal state throughout.

This must be the interpretation of the second theorem of $\S 24$. There is a theoretical possibility of failure, for it is possible (although infinitely improbable) that the value of $\mathrm{H}$ may differ from its minimum value by a finite amount through the whole of a stream-line. This question will be continued in a later section $(\$ 38)$.

$\S 32$. We now examine the third proposition of $\S 24$. If we consider all the points on the various stream-lines for a given value of $\epsilon$, which have a value of $\mathrm{H}$, say $\overline{\mathrm{H}}^{\prime}$, different from the minimum, we see (from the proposition analogous to (iii.) of $\$ 24$ ) that only for an infinitesimal fraction of these can $\mathrm{H}$ increase in either direction to a value of $\mathrm{H}$ which is greater by a finite amount than $\overline{\mathrm{H}}^{\prime}$. Thus of the points for which $\mathrm{H}=\overline{\mathrm{H}}^{\prime}$, this value of $\mathrm{H}$ is a maximum on the particular stream-line to which it belongs, for all except an infinitesimally small proportion of these stream-lines. Hence if we start a gas in any configuration for which $\mathrm{H}$ is greater than its minimum value, it is infinitely probable that in the initial motion $d \mathrm{H} / d t$ will be zero or negative.

Here we have the solution of the apparent paradox mentioned in $\S 17$. There is no real irreversibility in the motion of the gas on the whole, but there is an apparent irreversibility if we start from a point at which $\mathrm{H}$ is different from its minimum value. 


\section{Recapitulation and Discussion.}

$\S 33$. The whole of what has been proved amounts to the following : Firstly, a gas not in the normal state tends to approach that state; and, secondly, in examining the physical behaviour of a gas, departures from the normal state are insignificant, and we may legitimately proceed as if the gas were in the normal state throughout.

These results have only been obtained for the simplest type of gas, but it is obvious that they can be extended so as to apply to any kind of gas. The normal state is in each case found by assigning the minimum value to the function analogous to the $\mathrm{H}$-function already discussed-a function which may be conveniently referred to as Boltzmann's minimum-function.

The whole of the physical behaviour and statistical properties of a gas can be deduced from a knowledge of the H-function, just as the behaviour of a dynamical system can be deduced from a knowledge of the energy-function.

Some examples of the use of this function, illustrating some of the peculiarities which may arise, are given in the remaining sections :-

\section{Examples of the Minimum-theorem. \\ I.-Field of Eaternal Force.}

$\$ 34$. Let us suppose the molecules to move in a field of external force of potential $\chi$, a function of $x, y, z$. The analysis is exactly similar to that of $\S 29$, except that equation (37) must be replaced by

$$
\iiint \iiint\left\{\frac{1}{2} m\left(u^{2}+v^{2}+w^{2}\right)+\chi\right\} f d x d y d z d u d v d w=\mathrm{E}, . \text { ( }
$$

and from this we immediately obtain the law

$$
\begin{aligned}
& f=\mathrm{A} e^{-h m\left(u^{2}+v^{2}+w^{2}\right)-2 h} \chi . \quad . \quad . \\
& \text { 1I. - Mixture of Gases. }
\end{aligned}
$$

$\S 35$. Let the $\mathrm{N}$ molecules be ol different kinds, $\alpha \mathrm{N}$ of one kind, $\beta \mathrm{N}$ of another, and so on. The various coordinates can be represented in a generalized space as before. The chance that the whole gas shall be in a specified state is the product of the separate chances that the gas of each kind shall be in the corresponding state, whence we find, as the correct form for $\mathrm{H}$,

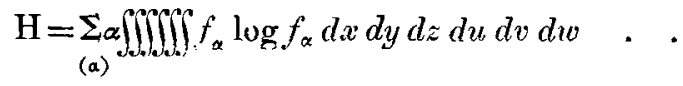


in which $f_{\alpha}$ is the law of distribution of the moleeules of the first kind, and so on. The conditions to which $f_{\alpha}, f_{\beta} \ldots$ are subject are, firstly, conditions of the form

$$
\iiint \iint_{.} f_{\alpha} d u d v d z v d x d y d z=1, \quad \text {. . . }
$$

and similar conditions for $f_{\beta}, \& c$, and, secondly, the single energy condition

$$
\sum_{(a)} a \iiint \iint_{0} \frac{1}{2} m_{\alpha}\left(u^{2}+v^{2}+w^{2}\right) f_{\alpha} d u d v d w d x d y d z=\mathrm{E} .
$$

The equations giving $f_{\alpha}, f_{\beta} \ldots$ are found to be

$$
1+\log f_{\alpha}+\lambda_{\alpha}+\frac{1}{2} \mu m_{\alpha}\left(u^{2}+v^{2}+w^{2}\right)=0,
$$

and this gives a solution of the form

$$
\begin{aligned}
& f_{a}=\mathrm{A} e-h m_{a}\left(u^{2}+v^{2}+w^{2}\right) \\
& f_{\beta}=\mathrm{B} e-h a n_{\beta}\left(u^{2}+v^{2}+w^{2}\right), \text { \&c. . . . }
\end{aligned}
$$

The constant $h$ is the same throughout, whence the BoyleCharles-Avogadro law can be deduced at once.

$$
\text { III.-Molecules of Finite Size. }
$$

$\S 36$. The analysis of $\S 27$ applies without alteration to this case, being independent of the size of the molecules. The analysis of $\$ \S 19-26$ also applies if a correction is made for the regions of the generalized space which are excluded by equations (3). It will be found that this correction has no effect on the ultimate result.

We also see from $\$ 13$ that the "chance of two molecules having assigned positions" is independent of the velocities of these molecules-a result which is in direct opposition to the views put forward by Burbury*.

$$
\text { IV.-The Law of Equipartition. }
$$

$\S 3 \pi$. The method of the present paper is in no way limited to the simple type of molecule which has been discussed up to the present. If each molecule has $m$ degrees of freedom we must suppose our generalized space to possess $2 m \mathrm{~N}$ dimensions, and we can proceed exactly as before. If $\mathrm{E}$ now denotes the energy of a single molecule, we find that no matter what function of the $2 \mathrm{~m}$ coordinates $\mathrm{E}$ may be, the law of distribution is of the form

$$
f=A e^{-2 k E} \quad . \quad . \quad . \quad . \quad .
$$

* ' Kinetic Theory of Gases,' chapters v. and vi. 
and the law of equipartition can be deduced in the usual way.

This proof of the law of equipartition is independent of the special assumptions both of Maxwell and of Boltzmann. We do not assume (as Maxwell does)

(a) That the representative point passes in turn through every point of that part of the generalized space for which the total energy has the assigned value.

(b) That the total time during which the point is in any element of this space is proportional to the size of the element.

Neither do we assume (as Boltzmann does)

(c) That the gas is, at every instant, "ungeordnet," or, more precisely, that Burbury's "Condition A" is satisfied at every instant.

The precise assumptions upon which our proof rests are:-

(d) That at any instant that part of the total energy of the gas which is accounted for by the intermolecular forces forms an infinitesimal fraction of the whole; and

(e) That the conservation of energy is maintained throughout the motion of the gas.

\section{V.-A Gas with a Mass-Velocity.}

$\S 38$. We have seen $(\$ 30)$ that a gas is in a state different from its normal state through only an inappreciable fraction of the whole of the generalized space, but at the same time $(\$ 31)$ it is possible that it may be in a state different from normal throughout the whole of a stream-line. For instance, the normal state is such that the mass-velocity of the gas is nil, but we know that if the gas starts with a mass-velocity it will retain this velocity throughout its motion, and will therefore never reach a normal state. The stream-lines for which the gas possesses an appreciable mass-velocity form only an inappreciable fraction of the whole, and are not worthy of consideration with reference to our arbitrary standard of probability. With reference to the conditions of nature the case is different, so that we now proceed to consider these particular stream-lines.

Let U, V, W be the components of the mass-velocity of the gas, then the analysis of $\$ 29$ will apply, provided we suppose $f$ subject not only to the conditions expressed by equations (36) and (37), but also to

$$
\iiint \iiint u f d x d y d z d u d v d v=\mathrm{U} \quad \text {. . . }
$$


and two similar equations for $\mathrm{V}$ and $\mathrm{W}$. The resulting law is found to be

$$
f=\mathrm{A} e^{-h m\left[(u-\mathrm{U})^{2}+(v-\mathrm{V})^{2}+(u-\mathrm{W})^{2}\right] .}
$$

The case of a mass-velocity of rotation may be treated in the same way.

$\$ 39$. We have so far dealt only with the case in which the energy is supposed to remain constant throughout the motion. Besides the energy there are no quantities which are known to remain constant throughout the motion except the six mass-velocities of rotation and translation, and, of course, the total number of molecules in the gas. For the purposes of the Kinetic Theory, we may suppose these massvelocities to each be zero. The solution is then given by equation (45).

On the other hand, the values obtained for the ratio of the specific heats show that this solution does not accord with the facts of nature. This may be for either of two reasons.

It may be that the number of constant quantities is greater than we have supposed, and that the knowledge of some now unknown constant would alter our result, just as $\$ 38$ a knowledge of the mass-relocity altered the result previously obtained. As I have suggested elsewhere, the uniformity of the experimental results obtained from different samples of gas supplies an argument of overwhelming strength against this supposition*.

Or it may be that the number of constant quantities is less than we have supposed, through the energy of the gas not remaining constant, a possibility which provides an escape from the difficulties in question.

LXV. On Oscillatory Discharges.

By A. BAtTelli and L. Magri $\dagger$.

[Plate XV.]

PART II. $\ddagger$

The Arrangement and General Course of Experiments.

30. DEFORE reporting the results of experiments, we $B$ wish to describe the whole arrangement of the apparatus.

* "The Condition necessary for Equipartition of Energy," Phil. Mag.

[6] iv. p. $585, \S \S 14-17$.

$\dagger$ Communicated by the Authors.

$\ddagger$ Part I., this volume, ante, pp. 1-34. 